Notfall Rettungsmed 2014 · 17:371-372

DOI 10.1007/s10049-014-1921-0

Online publiziert: 24. Juli 2014

๑) Springer-Verlag Berlin Heidelberg 2014

H. Moecke ${ }^{1} \cdot$ H. Müller ${ }^{2,3}$

${ }^{1}$ Konzernbereichsleiter Medizin und Wissenschaft, Asklepios Kliniken GmbH, Hamburg

${ }^{2}$ Aktionsbündnis Patientensicherheit APS, Berlin

${ }^{3}$ WINEG, Hamburg

\title{
Botschafter und Übersetzer gesucht
}

Diese Aufzählung solcher Beispiele ließe sich ohne Schwierigkeiten noch erweitern.

An vielen Krankenhäusern haben sich Teams gefunden, die das Thema Patientensicherheits- und Simulationstraining aufgegriffen haben.

Trotz dieser positiven Beispiele muss aber leider konstatiert werden, dass das Thema Patientensicherheits- und Simulationstraining noch keinen "Routineplatz“ im deutschen Gesundheitswesen gefunden hat. Woran liegt das? volles und bislang nicht ausreichend genutztes Instrument.

- In medizinischen Fachkreisen ist diese These inzwischen gut akzeptiert. Der 119. Deutsche Ärztetag hat sich damit 2013 beschäftigt, das Traumanetzwerk der DGU fordert es für die Zertifizierung, die S3-Leitlinie Sedierung in der Endoskopie sieht es vor und die DGAI fordert es als integralen Bestandteil der Weiterbildung, das APS für die Ausbildung. So haben das APS und der MDK Bayern mit weiteren Partnern das Projekt SimParTeam auf den Weg gebracht, um durch simulatorgestütztes Kreißsaaltraining die Sicherheit für Mutter und Kind unter der Geburt zu erhöhen. Mehrere Landesärztekammern haben das Simulatortraining in ihre Weitebildungsordnung aufgenommen. 25 der 50 geforderten Einsätze im Notarztdienst, die für die Zusatzweiterbildung Notfallmedizin gefordert werden, können im Simulatortraining abgeleistet werden.

\section{) Simulationstraining hat noch keinen "Routineplatz" im deutschen Gesundheitswesen gefunden}

In anderen Industrien, z. B. in der Energiewirtschaft, der Chemie, aber natürlich auch in der Luftfahrt, ist es Industriestandard, dass die Mitarbeiter, die komplexe Anlagen und Verfahren steuern, regelmäßig in einer Simulationsumgebung trainiert werden. Das wird nicht aus altruistischen Gründen gemacht. Diese Firmen sind überzeugt, dass das kostspielige und aufwendige Simulatortraining effizient, effektiv und wirtschaftlich sinnvoll für den Erfolg des Unternehmens ist.

Auch in der Medizin ist ein qualifiziertes und hochwertiges Patientensicherheits- und Simulationstraining mit nicht unerheblichen Kosten verbunden. Hohe Investitionen für die Simulationsumgebung, erheblicher Personalbe- darf an Trainern durch Kleingruppentraining und Abwesenheitskosten der Teilnehmer summieren sich. Diese Kosten sind in den meisten Fortbildungs- und Patientensicherheitsbudgets nicht eingeplant. Ohne auskömmliche Finanzierung ist das Training aber nicht realisierbar.

In unserem Gesundheitssystem brauchen wir deshalb viele Übersetzer und Botschafter, die die Sinnhaftigkeit und den wirtschaftlichen Nutzen von Patientensicherheits- und Simulationstrainings an die Entscheidungsträger im Gesundheitswesen überzeugend herantragen und damit dies bei Politik, Kostenträgern und Klinikführungen zu einem selbstverständlichen Element der Personalentwicklung und Sicherheitskultur machen, das entsprechend budgetiert ist. Patientensicherheits- und Simualtionstrainings bedürfen nicht zuletzt auch einer systematischen Finanzierung innerhalb des Gesundheitssystems.

Der verstärkte Einsatz von Simulationstechniken ist für den von allen Seiten geforderten Ausbau der Patienten-

Bei der Schlussredaktion dieser Ausgabe von Notfall+Rettungsmedizin erreichte uns die traurige Nachricht, dass Professor Dr. Stephen Abrahamson am 14. Juli 2014 im Alter von 93 Jahren verstorben ist. Mit der Entwicklung von Sim One im Jahr 1967 war er der Pionier in der Entwicklung von Patientensimulatoren. Gegen viele Widerstände entwickelte er das Konzept kontinuierlich weiter und legte so die Basis für das heutige Niveau des Patientensicherheits- und Simulationstrainings weltweit. In Dankbarkeit und mit großer Anerkennung nehmen wir Abschied. Möge er unvergessen bleiben. Seiner Familie und seinen Freunden kondolieren wir. 
sicherheit ethisch und gesundheitswissenschaftlich gefordert. Unser gemeinsamer Einsatz für das Patientensicherheits- und Simulationstraining wird in wenigen Jahren in Deutschland die Aus-, Weiter- und Fortbildung in den medizinischen Fachberufen sowie die Sicherheits- und Zusammenarbeitskultur in der Gesundheitswirtschaft insgesamt positiv weiterentwickeln.

Fangen wir alle mit dieser Überzeugungsarbeit noch heute an.

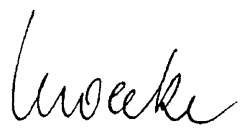

Prof. Dr. Heinzpeter Moecke

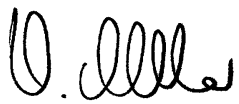

Hardy Müller

\section{Korrespondenzadresse}

\section{Prof. Dr. H. Moecke}

Konzernbereichsleiter Medizin

und Wissenschaft,

Asklepios Kliniken $\mathrm{GmbH}$

Hamburg

h.moecke@asklepios.com

\section{Einhaltung ethischer Richtlinien}

Interessenkonflikt. H. Moecke: Das Institut hat für Beratungstätigkeiten Unterstützung von LAERDAL MEDICAL erhalten. H. Müller hat keinen Interessenkonflikt.

\section{Rettungsdienst gegen Kindes- misshandlung}

Laut polizeilicher Kriminalstatistik versterben in Deutschland jede Woche drei Kinder infolge von Misshandlung und 70 Kinder bedürfen nach Misshandlung medizinischer Hilfe. Hierbei handelt es sich lediglich um die polizeilich bekannt gewordenen Fälle. Seriöse Schätzungen gehen davon aus, dass die Dunkelziffer der Kindesmisshandlung um einiges größer ist. Man vermutet das zwischen 200.000 und 1.440.000 Kinder jedes Jahr in Deutschland von Gewalt betroffen sind. Als Kindesmisshandlung im weiteren Sinne werden alle Erscheinungsformen von Gewalt gegen Kinder bezeichnet, wie körperliche Misshandlung, Vernachlässigung, psychische Misshandlung (z.B. Überforderung, seelische Grausamkeit) und sexueller Missbrauch. Im medizinischen Alltag begegnen dem Rettungsfachpersonal und den Notärzten am häufigsten die körperlichen Misshandlungen und die Vernachlässigungen. Daher muss der Rettungsdienst alles Erdenkliche tun, um diese Straftaten zu erkennen und zu melden. Was können Sie tun, wenn Sie in Ihrem Verantwortungsbereich Kinder sehen, auf die die oben genannten Befunde zutreffen?

- Bringen Sie das Kind, bei dem sie eine Kindesmisshandlung vermuten, in eine Klinik, damit hier in Ruhe die notwendige Diagnostik erfolgen kann. Es können, gerade beim Schütteltrauma, schwerste lebensbedrohliche Verletzungen vorliegen, die äußerlich überhaupt nicht feststellbar sind.

- Bei Hinweisen auf Vernachlässigung besteht bei Säuglingen immer Lebensgefahr und eine Klinikeinweisung ist obligat.

- Teilen Sie dem Klinikpersonal bei der Übergabe mit, wenn Sie ungewöhnliche oder verdächtige Beobachtungen gemacht haben, damit das Klinikpersonal diese in der Anamnese vermerken und somit auf Ihre Beobachtungen zurückgreifen und aufbauen kann.

- Vermerken Sie Ihre Beobachtungen und Befunde detailliert in der Einsatzdokumentation. Es kommt vor, dass Mitarbeiter des Rettungsdienstes im Strafverfahren teilweise Wochen, Monate oder manchmal auch Jahre später zu ihrem Einsatz, ihren Beobachtungen und/oder zu Angaben von Beteiligten befragt werden. Bei der Anzahl Ihrer Einsätze ist es da unwahrscheinlich, dass Sie dann noch alle Details im Kopf haben!
- Wenn Ihnen der Fall nahe gegangen ist, besprechen Sie ihn in ihrem Team.

- Sollten Sie über eine Meldung bei der Polizei nachdenken, besprechen Sie auch dieses im Team und/ oder mit Ihren Vorgesetzten! Die Möglichkeit zur anonymen Anzeige besteht immer. Sie könnten damit das Leben eines Kindes retten.

Mitarbeiter im Rettungsdienst unterliegen wie Ärzte gemäß § 203 StGB der Schweigepflicht. Die Schweigepflicht ist ein hohes Gut! Zur Abwehr einer unmittelbaren Gefahr für das Kind, sofern die Gefahr nicht anders abwendbar ist, hat der Gesetzgeber die Möglichkeit geschaffen, trotz fehlender Entbindung von der ärztlichen Schweigepflicht, das Jugendamt bzw. die Polizei zu informieren ( $\$ 34$ StGB).

Der Deutsche Berufsverband Rettungsdienst e.V. (DBRD) und das Institut für Rechtsmedizin der Charité - Universitätsmedizin Berlin wollen das Rettungsfachpersonal und die Notärzte unterstützen. Um eine schnelle Übersicht mit der Abgrenzung zwischen typischen Verletzungsmustern bei Unfällen und denen von Kindesmisshandlung zu bekommen, liegt dieser Ausgabe eine Taschenkarte bei.

Einen Folder mit weiteren Informationen finden Sie unter: http://www.dbrd.de/images/ aktuelles/2014/DBRD_Folder_Kindesmisshandlung.pdf 